\title{
Capecitabine pharmacogenetics: historical milestones and progress toward clinical implementation
}

\author{
"...in the past decade substantial progress has been made in the \\ recognition and understanding of the genetic underpinnings \\ of variability in capecitabine-related toxicity, which has led \\ to genotypic considerations being incorporated into dosing \\ recommendations."
}

First draft submitted: 28 July 2016; Accepted for publication: 28 July 2016; Published online: 27 September 2016

Keywords: capecitabine $\bullet$ DPYD $\bullet$ fluoropyrimidines $\bullet$ hand-foot syndrome $\bullet$ pharmacogenetics - thymidylate synthase $\bullet$ TYMS $\bullet$ Xeloda

The third-generation, orally-available fluoropyrimidine capecitabine (Xeloda ${ }^{\mathrm{TM}}$, Hoffmann-La Roche, Basel, Switzerland) has approved indications for the front-line therapy of advanced and/or metastatic colorectal cancer and pretreated metastatic breast cancer. Being a 5-fluorouracil prodrug, it has also increasingly substituted infusional fluorouracil as the fluoropyrimidine backbone in chemotherapy regimens for advanced esophagogastric malignancies on the basis of equivalent efficacy and its convenient outpatient oral dosing schedule [1-3]. Despite the proven efficacy and tolerability profile of capecitabine, selection of an optimal starting dosage remains a challenge, and clinical practices for treatment initiation differ worldwide [4]. Stark interindividual and inter-regional heterogeneity exists with respect to toxicity and efficacy profiles, and may be partially explained by genetic variation, with the most well-established pharmacogenes to-date being DPYD and $T Y M S$. In this Editorial, we highlight several historical milestones and recent progress that have added to the evidence base for clinical implementation of pharmacogenetics-guided capecitabine dosing.

To better appreciate the potential role of pharmacogenetics in personalized capecitabine chemotherapy, it seems apposite to take stock of its clinical development and postmarketing pharmacovigilance. The US FDA-approved marketing dosage of $1250 \mathrm{mg} / \mathrm{m}^{2}$ twice a day every 2 weeks on/1 week off (intermittent schedule) arguably has roots in an initial Phase I trial, which dose-escalated patients with a variety of solid tumors to capecitabine monotherapy based on the intermittent schedule [5], as well as a randomized Phase II study in advanced colorectal cancer where this schedule proved superior to two alternative dosing schedules in terms of time-to-progression (TTP; median TTP: 230 vs 127 and 165 days) and dose intensity [6]. However, early anecdotal accounts from US oncologists suggested that dose reductions may improve the tolerability of capecitabine without compromising its efficacy [7]. In 2005, a postmarketing retrospective review conducted at the MD Anderson Cancer Centre, which included 113 evaluable consecutive women with metastatic breast cancer, the majority (76\%) of whom were Caucasian [7], argued that a lower dosage of $1000 \mathrm{mg} / \mathrm{m}^{2}$ may be associated with a more favorable therapeutic index, as evidenced by reduced incidence of grade 3/4 toxic effects including hand-foot syndrome (20 vs $33 \%$ ), diarrhea (3 vs $13 \%$ ) and stomatitis (3 vs $8 \%$ ), and a lack of appreciable difference in response rates or TTP duration [7].

The improved therapeutic index of lowerdosage capecitabine among patients of Caucasian descent was supported in 2008 by a multinational, retrospective analysis of three large randomized controlled trials by Haller et al. in the Journal of Clinical Oncol-

\section{Nicholas Syn}

Department of Haematology-Oncology, National University Cancer Institute, Singapore 119228

and

Cancer Science Institute of Singapore, National University of Singapore, Singapore 117599 and

Department of Pharmacology, Yong Loo Lin School of Medicine, National University of Singapore, Singapore 117600

\section{Soo-Chin Lee}

Department of Haematology-Oncology, National University Cancer Institute,

Singapore 119228

and

Cancer Science Institute of Singapore,

National University of Singapore,

Singapore 117599

\section{Boon-Cher Goh}

Department of Haematology-Oncology, National University Cancer Institute,

Singapore 119228

and

Cancer Science Institute of Singapore,

National University of Singapore,

Singapore 117599

and

Department of Pharmacology, Yong Loo Lin School of Medicine, National University of Singapore, Singapore 117600

\section{Wei-Peng Yong}

Author for correspondence:

Department of Haematology-Oncology, National University Cancer Institute, Singapore 119228

and

Cancer Science Institute of Singapore,

National University of Singapore,

Singapore 117599

Tel.: +6567724140

Fax: +6567725680

Wei_Peng_YONG@nuhs.edu.sg

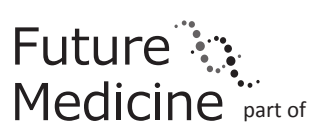


ogy [4], which was one of the first major reports to highlight the pharmacoethnicity of fluoropyrimidine agents. The analysis included a total of 3053 colorectal cancer patients from the USA, Europe and Asia who received capecitabine with or without oxaliplatin, or bolus 5-fluorouracil/leucovorin, and was adjusted for potential confounding factors (age, gender, treatment regimen and creatinine clearance). Confirming the anecdotal accounts, US patients were at significantly elevated risk for grade $3 / 4$ gastrointestinal adverse events (risk ratio: 3.62; 95\% CI: $2.11-6.20$; $\mathrm{p}<0.001$ ) and drug discontinuation (risk ratio: 1.84; $95 \% \mathrm{CI}$ : 1.14-2.96; $\mathrm{p}<0.001$ ) compared with patients from east Asia [4]. Furthermore, market data indicated that US physicians routinely initiate capecitabine at a lower dosage of $1000 \mathrm{mg} / \mathrm{m}^{2}$ on the intermittent schedule, further corroborating the notion that a lower dosage empirically produces a more favorable therapeutic index among Caucasian patients $[4,7]$.

"Compared with the hypothesis-driven candidate
gene approach, which has been the preferred
methodology in previous pharmacogenetic
analyses, the agnostic genome-wide association
study strategy would be tremendously helpful
for elucidating the complete pharmacogenetic
repertoire of capecitabine-related toxicities."

At the time, evidence that germinal polymorphisms in the metabolic and pharmacodynamic pathways of fluoropyrimidine drugs may modulate fluoropyrimidine toxicity risk was also emerging $[4,8,9]$. Schwab et al. reported in 2008 that several genetic polymorphisms, especially those in $D P Y D$, were highly predictive of severe fluorouracil toxicities [9]. Indeed, one of the best established genetic causes of fluoropyrimidine intolerance is deficiency of DPD, the rate-limiting enzyme involved in the catabolism of 5-fluorouracil that is encoded by the DPYD gene. Recent large metaanalyses have confirmed that the presence of several deleterious $D P Y D$ variants, including ${ }^{*} 2 A$, c. $2846 \mathrm{~A}>\mathrm{T}$, c. $1679 \mathrm{~T}>\mathrm{G}$ and c.1236G $>\mathrm{A} / \mathrm{HapB} 3$ is associated with severe toxic reactions to fluoropyrimidine drugs ([10-12] and references contained within). Both the Clinical Pharmacogenetics Implementation Consortium and Royal Dutch Association for the Advancement of Pharmacy-Pharmacogenetics Working Group have since embraced fluoropyrimidine dosing guidelines based on DPYD genotypes [13,14], and a recent Perspective in this journal espoused the possibility of using a $D P Y D$ gene activity score to tailor fluoropyrimidine dosages [15]. However, many of these DPYD variants are rare with a heterozygote frequency of about $1 \%$ or less among white people [10], which provides relatively low sensitivity to identify patients at risk of severe toxicity. For instance, the combined sensitivity of genotyping c. $1905+1 \mathrm{G}>\mathrm{A}, \quad$ c. $1601 \mathrm{G}>\mathrm{A}, \quad$ c. $1679 \mathrm{~T}>\mathrm{G}$ and c. $2846 \mathrm{~A}>\mathrm{T}$ for grade $3 / 4$ fluoropyrimidine-related toxicity is $23 \%$ (despite having a positive predictive value of $>99 \%$ and negative predictive value of $80 \%$ ) [16], underscoring the need to identify additional markers of fluoropyrimidine-related toxicity.

However, pharmacogenetic analyses were also focused on the major drug target of capecitabine, thymidylate synthase (TS), which is encoded by the TYMS gene. TS inhibition by the active metabolite fluorodeoxyuridine monophosphate prevents the formation of thymidylate (dTMP), which is a precursor for DNA synthesis, leading to cell-cycle arrest and apoptosis. Unlike DPYD, the TYMS gene harbors common functional variation, and the prominent interethnic variability in allelic frequencies mirrors inter-regional differences in capecitabine toxicity rates $[4,8]$. The TYMS promoter enhancer region (TSER) contains a variable number of 28-bp tandem repeats polymorphism, which usually occur in duplet (2R) and triplet (3R) forms, with the latter being more transcriptionally active. The $3 \mathrm{R}$ allele is predominant in east Asian populations and the $3 R / 3 R$ genotype is approximately twice as common among the Chinese population (67\%) compared with the Caucasian population (38\%) [17]. Retrospective analyses have reported grade $3 / 4$ toxicity rates of $43-63 \%, 18-32 \%$ and $3-27 \%$ in patients carrying the $2 R / 2 R, 2 R / 3 R$ and $3 R / 3 R$ genotypes, respectively, who received fluorouracil-based chemotherapy $[9,18]$, and a recent meta-analysis (five studies including 1303 patients) demonstrated that each additional copy of the $2 \mathrm{R}$ allele conveyed a $36 \%$ (95\% CI: $15-60 \%$; $\mathrm{p}<0.001$, fixed-effects model) increased odds of experiencing grade 3 or higher global capecitabine toxicity [12]. On the other, the $3 R / 3 R$ genotype appears to be associated with fluoropyrimidine resistance $[18,19]$, warranting the question whether patients with this genotype might stand to benefit from higher dose capecitabine.

As interethnic differences in the allelic frequencies of common TSER variants seemed to provide a compelling basis for the substantial inter-regional disparities in capecitabine toxicity and efficacy profiles observed $[4,8,9]$, we asked if it may be possible to tailor capecitabine dosages according to TSER genotype [18]. We, therefore, performed a TSER genotype-guided Phase I dose-escalation study to determine the maximum tolerable dose and recommended Phase II dose (RP2D) for patients with the TSER $3 \mathrm{R} / 3 \mathrm{R}$ or $2 \mathrm{R} /$ genotypes [18]. The classification of $2 R / 2 R$ and $2 R / 3 R$ into a single arm ( $2 \mathrm{R} /$ ) $)$ was planned a priori because the prevalence of these genotypes are uncommon in the 
Asian setting from which patients were recruited, and accrual was expected to be challenging. Capecitabine was dose-escalated in patients with advanced and/or metastatic solid tumors from 1250 to $1625 \mathrm{mg} / \mathrm{m}^{2}$ twice a day in intervals of $125 \mathrm{mg} / \mathrm{m}^{2}$ on the intermittent schedule, separately in TSER 3R/3R $(n=18)$ or $2 R / 2 R+2 R / 3 R(n=5)$ patients. With the stratification of patients by genotypes, the maximum tolerable dose and RP2D were declared at the 1625 and $1500 \mathrm{mg} / \mathrm{m}^{2}$ dose levels for $3 \mathrm{R} / 3 \mathrm{R}$ patients, which are considerably higher than the empirical dosage of $1000 \mathrm{mg} / \mathrm{m}^{2}$ that is frequently prescribed by US practitioners $[4,7,18]$. However, dosage recommendations for patients with the TSER 2R/_ genotypes could not be determined due to poor accrual. It is worth noting that whereas most dose modifications in the oncological sphere typically entail a dose reduction, the study proposes that a subset of patients with the $3 \mathrm{R} / 3 \mathrm{R}$ genotype may in fact be amenable to a dose increment. Nonetheless, the clinical activity of a higher capecitabine dosage compared with the approved dosage for TSER 3R/3R patients remains to be evaluated in a Phase II study.

In summary, in the past decade substantial progress has been made in the recognition and understanding of the genetic underpinnings of variability in capecitabine-related toxicity, which has led to genotypic considerations being incorporated into dosing recommendations [13-15,18]. However, cautious optimism with respect to the clinical utility of pre-emptive pharmacogenetic testing is warranted: an SNP panel comprising well-established DPYD and TYMS variants only provides $26 \%$ sensitivity, $86 \%$ specificity and $49 \%$ positive predictive value, which would be suboptimal to inform routine clinical decisionmaking [12]. Evidently, much work remains to identify and validate additional markers of capecitabine toxicity. Compared with the hypothesis-driven candidate gene approach, which has been the preferred methodology in previous pharmacogenetic analy-

\section{References}

1 Midgley R, Kerr DJ. Capecitabine: have we got the dose right? Nat. Clin. Pract. Oncol. 6(1), 17-24 (2009).

2 Cunningham D, Starling N, Rao S et al. Capecitabine and oxaliplatin for advanced esophagogastric cancer. N. Engl. J. Med. 358(1), 36-46 (2008).

3 Kang Y-K, Kang W-K, Shin D-B et al. Capecitabine/ cisplatin versus 5 -fluorouracil/cisplatin as first-line therapy in patients with advanced gastric cancer: a randomised Phase III noninferiority trial. Ann. Oncol. 20(4), 666-673 (2009).

4 Haller DG, Cassidy J, Clarke SJ et al. Potential regional differences for the tolerability profiles of fluoropyrimidines. J. Clin. Oncol. 26(13), 2118-2123 (2008). ses [9-12,16], the agnostic genome-wide association study strategy would be tremendously helpful for elucidating the complete pharmacogenetic repertoire of capecitabine-related toxicities. A prospective genomewide association study of breast cancer patients treated with single-agent capecitabine from Singapore will soon be reported [20] and will reveal the extent to which common and rare variation modulates the risk of hand-foot syndrome, a common disfiguring dermatologic toxic reaction to capecitabine. Another area for consideration in future and ongoing studies is to prioritize patients who received capecitabine monotherapy (as opposed to capecitabine-containing combination regimens or who received other fluoropyrimidine drugs), as this would allow more accurate delineation of the pharmacogenetic associations with capecitabine toxicity. In fact, the clinical relevance of pharmacogenetics in capecitabine-containing regimens, where the dosage of capecitabine and the risk of acute side effects is lower, should be investigated. Finally, in selecting an appropriate dosage of capecitabine for an individual, it is important to also consider nongenetic factors such as dietary folate intake, as well as clinicopathological characteristics such as hepatorenal function, gender and age $[1,8,9]$. Resolution of these issues will be crucial to unlock the full potential of pharmacogenetics in personalized capecitabine chemotherapy.

\section{Financial \& competing interests disclosure}

WP Yong is supported by the National Medical Research Council (NMRC) Translational \& Clinical Research (TCR) Flagship Program (NMRC/TCR/009-NUHS/2013). The authors have no other relevant affiliations or financial involvement with any organization or entity with a financial interest in or financial conflict with the subject matter or materials discussed in the manuscript apart from those disclosed.

No writing assistance was utilized in the production of this manuscript.

5 Mackean M, Planting A, Twelves C et al. Phase I and pharmacologic study of intermittent twice-daily oral therapy with capecitabine in patients with advanced and/or metastatic cancer. J. Clin. Oncol. 16(9), 2977-2985 (1998).

6 Van Cutsem E, Findlay M, Osterwalder B et al. Capecitabine, an oral fluoropyrimidine carbamate with substantial activity in advanced colorectal cancer: results of a randomized Phase II study. J. Clin. Oncol. 18(6), 1337-1345 (2008).

7 Hennessy BT, Gauthier AM, Michaud LB et al. Lower dose capecitabine has a more favorable therapeutic index in metastatic breast cancer: retrospective analysis of patients treated at M. D. Anderson Cancer Center and a review of capecitabine toxicity in the literature. Ann. Oncol. 16(8), 1289-1296 (2005). 
8 Syn NL-X, Yong W-P, Lee S-C et al. Genetic factors affecting drug disposition in Asian cancer patients. Expert Opin. Drug Metab. Toxicol. 11(12), 1879-1892 (2015).

9 Schwab M, Zanger UM, Marx C et al. Role of genetic and nongenetic factors for fluorouracil treatment-related severe toxicity: a prospective clinical trial by the German 5-FU Toxicity Study Group. J. Clin. Oncol. 26(13), 2131-2138 (2008).

10 Meulendijks D, Henricks LM, Sonke GS et al. Clinical relevance of $D P Y D$ variants c.1679T $>\mathrm{G}, \mathrm{c} .1236 \mathrm{G}>\mathrm{A} / \mathrm{HapB} 3$, and $c .1601 \mathrm{G}>\mathrm{A}$ as predictors of severe fluoropyrimidineassociated toxicity: a systematic review and meta-analysis of individual patient data. Lancet Oncol. 16(16), 1639-1650 (2015).

11 Terrazzino S, Cargnin S, Del Re M et al. DPYD IVS14+1G $>A$ and $2846 \mathrm{~A}>\mathrm{T}$ genotyping for the prediction of severe fluoropyrimidine-related toxicity: a meta-analysis. Pharmacogenomics 14(11), 1255-1272 (2013).

12 Rosmarin D, Palles C, Church D et al. Genetic markers of toxicity from capecitabine and other fluorouracil-based regimens: investigation in the QUASAR2 study, systematic review, and meta-analysis. J. Clin. Oncol. 32(10), 1031-1039 (2014).

13 Caudle KE, Thorn CF, Klein TE et al. Clinical Pharmacogenetics Implementation Consortium guidelines for dihydropyrimidine dehydrogenase genotype and fluoropyrimidine dosing. Clin. Pharmacol. Ther. 94(6), 640-645 (2013).
14 Swen JJ, Nijenhuis M, de Boer A et al. Pharmacogenetics: from bench to byte - an update of guidelines. Clin. Pharmacol. Ther. 89(5), 662-673 (2011).

15 Henricks LM, Lunenburg CATC, Meulendijks D et al. Translating DPYD genotype into DPD phenotype: using the DPYD gene activity score. Pharmacogenomics 16(11), 1277-1286 (2015).

16 Loganayagam A, Arenas Hernandez M, Corrigan A et al. Pharmacogenetic variants in the DPYD, TYMS, CDA and MTHFR genes are clinically significant predictors of fluoropyrimidine toxicity. Br. J. Cancer 108(12), 2505-2515 (2013).

17 Marsh S, Collie-Duguid ESR, Li T et al. Ethnic variation in the thymidylate synthase enhancer region polymorphism among Caucasian and Asian populations. Genomics 58(3), 310-312 (1999).

18 Soo RA, Syn N, Lee S-C et al. Pharmacogenetics-guided Phase I study of capecitabine on an intermittent schedule in patients with advanced or metastatic solid tumours. Sci. Rep. 6, 27826 (2016).

19 Marsh S, McKay JA, Cassidy J, McLeod HL. Polymorphism in the thymidylate synthase promoter enhancer region in colorectal cancer. Int. J. Oncol. 19(2), 383-386 (2001).

20 Yap YS, Kwok L-L, Ng RCH et al. Predictors of hand-foot syndrome (HFS) in randomised double-blind, placebocontrolled trial of pyridoxine for prevention of capecitabine induced HFS. ASCO Meet. Abstr. 33, 9596 (2015). 